\title{
Differential subcellular localization and activity of kelch repeat proteins KLHDC1 and KLHDC2
}

King-Tung Chin', Hai-Tao Xu ${ }^{1,2}$, Yick-Pang Ching ${ }^{1,2}$, Dong-Yan Jin',

Departments of 'Biochemistry and 'Pathology, The University of Hong Kong, 21 Sassoon Road, Hong Kong, China

${ }^{3}$ Address for offprints: D.-Y. Jin, Department of Biochemistry, The University of Hong

Kong, 3/F, Laboratory Block, Faculty of Medicine Building, 21 Sassoon Road, Hong Kong.

Tel: $+852-2819-9491$

Fax: $+852-2855-1254$

E-mail: dyjin@hkucc.hku.hk 


\begin{abstract}
We have previously identified and characterized human KLHDC2/HCLP-1, a kelch repeat protein that interacts with and inhibits transcription factor LZIP. In this study we identified and characterized a paralog of KLHDC2 called KLHDC1. KLHDC1 and KLHDC2 share about $50 \%$ identity at the level of amino acid sequence and both gene loci localize to human chromosome 14q21.3. This cluster of KLHDC1 and KLHDC2 genes is highly conserved in vertebrates ranging from pufferfish to human. Both genes are expressed highly in skeletal muscle, but weakly in various other tissues. While KLHDC2 was predominantly found in the nucleus, KLHDC1 is a cytoplasmic protein. Neither KLHDC1 nor KLHDC2 binds to actin. In addition, KLHDC1 was unable to inhibit LZIP/CREB3-mediated transcriptional activation. Thus, KLHDC1 and KLHDC2 have differential localization and activity in cultured mammalian cells.
\end{abstract}

Key words: kelch repeat protein, KLHDC1, KLHDC2, transcription factor, LZIP Abbreviation: BD, Gal4 DNA binding domain; HCA33, hepatocellular carcinomaassociated antigen 33; HCF, host cell factor 1; HCLP, HCF-like protein; HUGO, The Human Genome Organization; KLHDC, kelch domain containing protein 


\section{Introduction}

The kelch motif of about 50 amino acids in length was first identified in fruit fly kelch ORF1 protein [1] and has subsequently been found in many different polypeptide contexts [2]. It commonly occurs in multiple repeats to form a tertiary structure known as $\beta$-propeller, each blade of which has four-stranded antiparallel $\beta$ sheets radiating outward from a central axis [3]. The kelch repeats are thought to be an evolutionarily conserved module that mediates protein-protein interaction [2]. Many kelch repeat proteins are critically involved in cytoskeletal organization through their association with actin and intermediate filaments [4]. Other kelch repeat proteins participate in diverse physiological processes including cell signaling, protein trafficking and transcriptional regulation [5].

Host cell factor 1 ( $\mathrm{HCF} 1)$ is the prototype of a group of multifunctional transcriptional regulators that have six kelch repeats at the N-terminus [6, 7]. HCF1 coordinates the orderly assembly of enhancer complexes on viral and cellular promoters through the interaction between its kelch domain and various transactivators such as LZIP, VP16, GA binding protein and Oct-1 [8-11]. The kelch repeats of HCF1 have also been found to associate with Set1/Ash2 methyltransferase [12]. They are thought to tether HCF1 to the chromatin and they also play an important role in the regulation of cell cycle progression and cell proliferation $[13,14]$. We have previously identified and characterized a novel protein designated HCF-like protein 1 (HCLP-1). HCLP-1 shares significant homology with HCF1 and HCF2. However, unlike HCF that has multiple functional domains, HCLP-1 is composed entirely of six kelch repeats [15]. When HCLP-1 was identified, the only other kelch repeat protein known to consist entirely of 
kelch repeats is p40, which binds to Rab9-GTP and is required for endosome-to-transGolgi network transport [16]. HCLP-1 specifically interacts with bZIP transcription factor LZIP but not with VP16. This interaction targets the bZIP domain and leads to an inhibition of LZIP-dependent transcription [15]. Because LZIP and other bZIP transcription factors in the CREB3 subfamily have tumor suppressive activity $[17,18]$, expression of HCLP-1 may influence cell proliferation through the inhibition of LZIP. Indeed, HCLP-1 has independently been identified as hepatocellular carcinomaassociated antigen 33 (HCA33; GenBank AF244137), which is abundantly found in hepatoma tissues. Because HUGO Gene Nomenclature Committee has subsequently named HCLP-1/HCA33 as kelch domain containing protein 2 (KLHDC2), thereafter in this paper we will use this official name.

In this study, we identified and characterized a novel kelch repeat protein KLHDC1 paralogous to KLHDC2. Interestingly, both KLHDC1 and KLHDC2 mapped to human chromosome 14q21.3. The two gene loci are found in head-to-tail orientation, forming a small gene cluster. We compared the amino acid sequences of the two proteins and the genomic organization of the two genes. In addition, we also examined the expression and localization patterns as well as the activity profile of KLHDC1 and KLHDC2. Our findings have important implications on the evolution and functional diversification of vertebrate kelch repeat proteins. 


\section{Experimental procedures}

\section{Plasmids}

Reporter plasmid pGal-Luc and expression plasmids for V5-tagged KLHDC2, Ga14 DNA binding domain (BD) and BD-LZIP fusion protein have previously been described [15]. Expression plasmid for V5-tagged KLHDC1 was derived from pcDNA3.1/V5/HisB (Invitrogen). Expression plasmids for BD-VP16-KLHDC1 and BD cJun-KLHDC1 were derived from $\mathrm{pM}(\mathrm{CLONTECH})$.

KLHDC1 cDNA (IMAGE clone 5283778; GenBank BC031270) was obtained from RZPD Deutsches Ressourcenzentrum für Genomforschung Gmbh (Berlin, Germany). Nucleotide A at position 560 found to be different from human/chimpanzee genomic sequence and from other KLHDC1 cDNA clones was corrected to G using site-directed mutagenesis and confirmed by DNA sequencing. Full-length cJun cDNA has been described elsewhere [15]. VP16 cDNA [19] was kindly provided by Dr. Gary Hayward (Johns Hopkins University at Baltimore, USA).

Details of KLHDC1/KLHDC2 expression plasmids and oligonucleotide primers used in subcloning are available upon request.

\section{Sequence analysis}

Multiple alignment of KLHDC1-related protein sequences was produced with the PILEUP program in the Wisconsin software package (Version 10.2; Genetics Computer Group, Inc., Madison, Wisconsin, USA) and the BOXSHADE server (Version 3.21; URL: 
http://www.ch.embnet.org). Phylogeny of protein sequences was inferred using the PHYLIP software package (Version 3.63; Joseph Felsenstein, The University of Washington) as previously described [20].

\section{Northern blot analysis}

Human multiple tissue Northern blots were purchased from CLONTECH. Northern blot analysis was performed using the protocol recommended by the manufacturer. The probes for Northern blotting were full-length cDNAs of human KLHDC1 $(2.7 \mathrm{~kb})$ and KLHDC2 $(1.7 \mathrm{~kb})$, while human $\beta$-actin cDNA $(2 \mathrm{~kb})$ was used as a control for equal loading of RNA samples.

\section{Luciferase assay}

Dual-luciferase assay was performed with extracts of transiently transfected HeLa cells as described elsewhere [21, 22]. Both firefly and sea pansy luciferase reporter enzymes were simultaneously expressed in the transfected cells. The activity of sea pansy enzyme was used to normalize the readouts of firefly luciferase activity.

\section{Confocal microscopy}

HeLa cells were cultured and transfected as previously described [18, 23]. Laserscanning confocal immunofluorescence microscopy was carried out as detailed elsewhere $[23,24]$. Images were captured at $63 \times$ magnification on MRC1024 system (BioRad) with the help of the LaserSharp software. For leptomycin B treatment, HeLa cells were incubated with leptomycin B at a final concentration of $15 \mathrm{~nm}$ for 5 hours. 
Mouse monoclonal anti-V5 antibody was purchased from Invitrogen. Mouse monoclonal anti-BD antibody (clone RK5C1) was from Santa-Cruz.

\section{Co-immunoprecipitation}

MCF-7 cells grown to about $70 \%$ confluence in $10-\mathrm{cm}$ dishes were transfected with KLHDC1- or KLHDC2-expressing plasmid. After 24 hours cells were lysed in HEPES lysis buffer [50 mM HEPES ( $\mathrm{pH} 7.5$ ), 1\% Nonidet P-40, 0.5\% sodium deoxycholate, 150 $\mathrm{mM} \mathrm{NaCl}, 1 \mathrm{mM}$ EDTA, $0.01 \%$ Triton X-100 plus protease inhibitor cocktails]. For each transfection the cell lysate was immunoprecipitated with $1 \mu \mathrm{l}$ anti-V5 antiserum as previously described [18].

Rabbit polyclonal anti-V5 antiserum and mouse monoclonal anti-actin antibody (clone AC-40) were purchased from Sigma. 


\section{Results}

KLHDC1 and KLHDC2 represent two subfamilies of kelch repeat proteins in vertebrates

The characterization of human KLHDC2 [15] facilitates the cloning of its orthologs and paralogs. On one hand, a comparison with the amino acid sequence of KLHDC2 led to the identification of mouse and rat orthologs. More than $95 \%$ of the 406 amino acid residues in murine Klhdc2 are identical to those in the human counterpart. In addition, chicken Klhdc2 and pufferfish klhdc2, which share $84 \%$ and $58 \%$ identity with human KLHDC2, respectively, were identified from the working draft of their genome sequences. On the other hand, we also found a novel human kelch repeat protein closely related to KLHDC2. This protein was subsequently named as KLHDC1 by the HUGO Gene Nomenclature Committee.

We assembled full-length KLHDC1 cDNA from human EST clones homologous to KLHDC2. The human KLHDC1 sequence was determined and a complete coding region was identified. KLHDC1 and KLHDC2 share $47 \%$ identity and $64 \%$ similarity in an overlap of 352 amino acid residues (Fig. 1). Both proteins consist entirely of six kelch repeats that form a $\beta$-propeller. Notably, orthologs of KLHDC1 were also found in mouse, rat and other vertebrates. For example, the mouse Klhdc1 and pufferfish klhdc1 share $85 \%$ and $51 \%$ identity with the human protein. Because a homology search in the current databases yielded additional KLHDC1/2-related sequences in vertebrates, we generated a consensus parsimony tree of representative KLHDC1/2 homologs from various vertebrate species (Fig. 2) in order to understand the genetic relationship.

In this phylogenetic tree, human KLHDC1 clusters with murine and other vertebrate 
Klhdc1 proteins, while KLHDC2 groups with its vertebrate orthologs (Fig. 2). The bootstrap supports for these clusters are statistically significant (97-99\%). While each vertebrate species had two KLHDC1/2 homologs, KLHDC1/2 orthologs were not found in invertebrates and other lower organisms. Thus, KLHDC1 and KLHDC2 likely represent two closely-related vertebrate subfamilies of kelch repeat proteins. It will be of interest to compare and contrast the biological activities of KLHDC1 and KLHDC2.

\section{Chromosomal location and genomic organization of KLHDC1/2 loci}

Chromosomal map locations of KLHDC1 and KLHDC2 loci in human genome Build 35.1 were determined by sequence alignment. Using cDNA information we identified genomic clones that contain the KLHDC1 and KLHDC2 loci. Interestingly, both loci map to chromosome 14q21.3, forming a small gene cluster, in which KLHDC1 and KLHDC2 are arranged in a head-to-tail orientation (Fig. 3A). The human KLHDC1/2 genes were noted to be closely linked to the loci of DNA polymerase $\varepsilon 2$ (POLE2) and serologically defined colon cancer antigen 1 (SDCCAG1). The distance between KLHDC1 and KLHDC2 is within $15 \mathrm{~kb}$. Notably, the map locations of Klhdc1 and Klhdc2 genes in mouse genome Build 34.1 are highly conserved (Fig. 3B). In the genome of pufferfish Tetraodon nigroviridis, while klhdc1 and klhdc2 loci are also adjacent to each other and closely linked to pole2 and sdccag1 loci, the order and orientation of genes are different (Fig. 3C). In particular, pufferfish pole2 locus lies between klhdc1 and klhdc2, which transcribe towards opposite directions. The high degree of conservation at the levels of amino acid sequence and of genomic organization suggests that the separation of klhdc1 and klhdc2 genes was an early event during the evolution of vertebrates. 
Human KLHDC1 locus spans $60 \mathrm{~kb}$, whereas mouse Klhdc1 and pufferfish klhdc1 are $42 \mathrm{~kb}$ and $3 \mathrm{~kb}$ in length, respectively (Fig. 3). However, the sizes of exons in KLHDC1 and KLHDC2 genes are relatively conserved in human, mouse and pufferfish genomes (Fig. 3D and Fig. 3E). The genes contain 12-13 exons varying in size from 53 bp to $1.6 \mathrm{~kb}$. Many exons are of the same size in all three species.

Expression of KLHDC1 and KLHDC2 transcripts in human tissues

To assess the expression pattern of KLHDC1 and KLHDC2 mRNA in human tissues, we performed Northern blotting (Fig. 4). Both KLHDC1 and KLHDC2 were abundantly expressed in skeletal muscle (Fig. 4A and Fig. 4B, lane 3). A relatively high level of KLHDC2 mRNA was also found in heart (Fig. 4B, lane 2). Meanwhile, weak expression of KLHDC1 and KLHDC2 transcripts was detected in most other tissues. However, they were apparently absent in peripheral blood leukocytes (PBL; Fig. 4A and Fig. 4B, lane 12). In consistent with our unpublished data, previous reports $[15,25]$ and microarray analysis (http://www.genecards.org) indicated that KLHDC1 and KLHDC2 are also expressed at moderate-to-high levels in pancreas and liver. Taken together, both KLHDC1 and KLHDC2 are ubiquitously expressed in human tissues albeit in low abundance. The similar expression patterns of KLHDC1 and KLHDC2 suggest that they might serve related function in vivo.

\section{Differential activity of KLHDC1 and KLHDC2 in the inhibition of LZIP}

We have previously shown that KLHDC2 inhibits LZIP-mediated transcription through direct interaction with LZIP [15]. To compare the influence of KLHDC1 and KLHDC2 
on LZIP-dependent transcription, we performed luciferase reporter assays. We noted that the expression of either KLHDC2 or KLHDC1 did not affect the basal transcriptional activity in BD-expressing HeLa cells (Fig. 5, columns 1-3). Consistent with our previous finding [15], the co-expression of KLHDC2 with BD-LZIP led to substantial inhibition of LZIP-dependent expression of the luciferase reporter (Fig. 5, compare column 5 to column 4). In contrast, the expression of KLHDC1 had no influence on LZIP-mediated activation of the luciferase gene (Fig. 5, compare column 6 to column 4). Hence, human KLHDC1 and KLHDC2 have differential LZIP-inhibiting activity in cultured cells. KLHDC1 is incapable of suppressing LZIP-dependent transcription.

\section{Differential subcellular localization of KLHDC1 and KLHDC2}

Above we have demonstrated the differential activity of KLHDC1 and KLHDC2 in the inhibition of LZIP (Fig. 5). To further investigate the underlying mechanism, we next queried for the subcellular localization of KLHDC1. We have previously shown that KLHDC2 localizes predominantly to the nucleus, where it interacts with and inhibits the active form of LZIP [15]. In this study, we first verified the nuclear localization of V5tagged KLHDC2 using an anti-V5 antibody (Fig. 6, panels A-C). Significant overlapping of KLHDC2-specific fluorescent signal with propidium iodide staining (Fig. 6, panel C) indicates that KLHDC2 is indeed a nuclear protein. In contrast, KLHDC1 was apparently found in the nucleus-excluded region (Fig. 6, panels D-F). The cytoplasmic localization of KLHDC1 was further corroborated by the staining patterns of two artificially constructed proteins fused to Gal4 DNA binding domain (BD): BD-VP16-KLHDC1 and BD-cJun-KLHDC1 (Fig. 6, panels G-L). BD contains a nuclear localization signal and is 
ambiently found in the nucleus [26]. VP16 and c-Jun are potent transcription factors and constitutively localize to the nucleus $[27,28]$. In fact they are usually capable of driving their fusion or dimerization partners efficiently into the nucleus [26, 28]. However, both BD-VP16-KLHDC1 and BD-cJun-KLHDC1 fusions localized predominantly to the cytoplasm (Fig. 6, panels G-L), suggesting that the KLHDC1 moiety in these proteins can sufficiently prevent BD-VP16 and BD-cJun from entering the nucleus. In further support of this, the addition of KLHDC1 to either N or C terminus of VP16 or c-Jun resulted in cytoplasmic retention of the fusion protein (data not shown). Plausibly, KLHDC1 is sequestered in the cytoplasm by as-yet unidentified partner protein(s).

We were unable to identify nuclear import and export signals in KLHDC1 or KLHDC2, but we could not rule out the possibility that more efficient or rapid export of KLHDC1 might account for its cytoplasmic localization. To compare the nuclear retention properties of KLHDC1 and KLHDC2, we treated KLHDC2- and KLHDC1expressing cells with leptomycin B, an inhibitor of CRM1-medaited nuclear export. If KLHDC1 is exported more rapidly from the nucleus in a CRM1-dependent manner, treatment with leptomycin B would block this export and lead to nuclear retention of the protein. On the other hand, if the distribution of KLHDC1 in the nucleus and the cytoplasm is not influenced by leptomycin B, CRM1-dependent nuclear export is unlikely a critical factor in determining the cytoplasmic localization of KLHDC1. We observed that the treatment of KLHDC2-expressing cells with leptomycin B led to the disappearance of residual KLHDC2-specific fluorescent signal in the cytoplasm (Fig. 7, compare panel D to panel A). In sharp contrast, the cytoplasmic staining pattern of KLHDC1 did not alter after leptomycin B treatment (Fig. 7, panel J compared to panel G). 
These results suggest that KLHDC1 is not substantially imported into the nucleus. This is consistent with our model that KLHDC1 is retained in the cytoplasm by its partner protein(s).

Neither KLHDC1 nor KLHDC2 associates with actin in vivo

The high abundance of KLHDC1 and KLHDC2 in skeletal muscles (Fig. 4) raises an interesting question as to whether these two kelch repeat proteins may have a specific function in muscle cells. Because many kelch repeat proteins associate with actin and modulate actin cytoskeleton $[2,5]$, it will be of interest to see whether KLHDC1 and KLHDC2 might interact with actin. In this regard, differential affinity to actin might also cause cytoplasmic retention of KLHDC1 as seen in Fig. 6 and Fig. 7. Thus, we compared the actin-binding activities of KLHDC1 and KLHDC2 by co-immunoprecipitation (Fig. 8). While both $\beta$-actin and KLHDC1/2 are abundant in the cell lysates, $\beta$-actin was not found in the anti-V5 immunoprecipitates containing KLHDC1 or KLHDC2 (Fig. 8A, lanes 6 and 8 compared to lanes 5 and 7, respectively). Hence, neither KLHDC1 nor KLHDC2 associates with actin in cultured MCF7 cells. 


\section{Discussion}

In this study we identified a novel kelch repeat protein called KLHDC1 and we compared the sequence, expression, subcellular localization and activity of KLHDC1 and KLHDC2. KLHDC1 is strikingly homologous to KLHDC2, a nuclear inhibitor of LZIP transcription factor (Fig. 1). Both KLHDC1 and KLHDC2 loci mapped to the same gene cluster on chromosome 14q21.3 and they situated next to each other in a head-to-tail orientation (Fig. 3). Orthologs of KLHDC1 and KLHDC2 were commonly found in other vertebrates. Phylogenetic analysis suggests that they represent two closely-related subfamilies of proteins composed entirely of kelch repeats (Fig. 2). KLHDC1 and KLHDC2 transcripts were abundantly expressed in skeletal muscles, and they were also found in low abundance in many other human tissues, except that high level of KLHDC2 mRNA was expressed in heart (Fig. 4). While the sequence and genomic structure of KLHDC1 and KLHDC2 genes are highly conserved, KLHDC1 and KLHDC2 showed differential activity in the inhibition of LZIP transcription factor (Fig. 5) and localized to different subcellular compartments (Fig. 6 and Fig. 7). Neither KLHDC1 nor KLHDC2 associates with actin in cultured cells (Fig. 8), but KLHDC1 is likely sequestered in the cytoplasm by unidentified partner protein(s). In contrast to KLHDC2 which is predominantly in the nucleus and potently inhibits LZIP-dependent transcriptional activity [15], KLHDC1 is primarily a cytoplasmic protein (Fig. 6 and Fig. 7) that has no influence on LZIPmediated transcriptional activation (Fig. 5).

The kelch repeats represent one of commonest modules that mediate protein-protein interactions in eukaryotes [2]. Although kelch domains adopt similar tertiary structures known as $\beta$-propeller, they are remarkably versatile in partner selection and binding [2]. 
Kelch repeat proteins are numerous and ancient $[2,5]$. While other kelch repeat proteins are found in organisms ranging from yeast to human, KLHDC1 and KLHDC2 exist only in vertebrates and they belong to a subfamily of kelch repeat proteins consisting entirely of $\beta$-propeller structure. This subfamily is only seen in animals. The highly conserved organization of KLHDC1 and KLHDC2 loci in vertebrate genomes implicates that they might probably be generated by duplication of a primordial gene in the ancestor of vertebrate animals. The expansion of the family of kelch repeat proteins in multicellular animals has been documented previously $[2,29]$. This expansion is accompanied with the emergence of kelch repeat proteins that are critically involved in cell signaling and transcriptional regulation [5]. In light of this, it will be of particularly great interest to elucidate the physiological roles of KLHDC1 and KLHDC2.

It is noteworthy that the $\beta$-propeller domain of KLHDC1 and KLHDC2 is particularly similar to that of HCF1 and HCF2 [15]. Our previous work demonstrates that KLHDC2 interacts with transcription factor LZIP [15], in a manner similar to the proteolytic fragments of HCF1 that contain the kelch domain only [30, 31]. Currently we do not understand the molecular basis of the differential activity of KLHDC1 and KLHDC2. However, these two proteins resemble another pair of human kelch repeat proteins HCF1 and HCF2, which have shown different subcellular localization and activity in cultured cells [5]. While HCF1 is an exclusively nuclear protein, HCF2 can be found predominantly in the cytoplasm [32]. In addition, whereas HCF1 efficiently binds with and coactivates VP16 and Oct1, HCF2 binds to these transcription factors inefficiently and it does not support their transcriptional activity [32, 33]. 
LZIP and closely related transcription factors normally reside in the endoplasmic reticulum and undergo nuclear translocation upon proteolytic activation $[18,34,35]$. KLHDC2 interacts with the activated form of LZIP in the nucleus and inhibits its transcriptional activity [15]. As a predominantly cytoplasmic protein, KLHDC1 has no chance to interact with and inhibit active LZIP in the nucleus. This might explain the inability of KLHDC1 to down-regulate LZIP-dependent transcription (Fig. 5). While we did not detect a direct interaction between KLHDC1 and LZIP (data not shown), we still could not rule out the possibility that KLHDC1 could bind to other transcription factors of the same subfamily [18] in the cytoplasm.

The kelch domains are thought to provide various contact sites for protein-protein interaction. One important route towards characterizing the physiological roles of KLHDC1 and KLHDC2 is to identify their protein partners. In particular, it will be of importance to compare the spectra of KLHDC1 and KLHDC2 partners obtained through yeast two-hybrid screening or co-immunoprecipitation. The characterization of these protein partners will likely derive additional mechanistic insight into the differential subcellular localization and activity of KLHDC1 and KLHDC2. 


\section{Acknowledgments}

We thank G.S. Hayward for gift of plasmid and A.C.S. Chun for critical reading of the manuscript. D.-Y. Jin is a Leukemia and Lymphoma Society Scholar. This work was supported by a grant to D.-Y. Jin from the Hong Kong Research Grants Council (Project HKU 7294/02M). 


\section{References}

1. Xue F, Cooley L: kelch encodes a component of intercellular bridges in Drosophila egg chambers. Cell 72: 681-693, 1993

2. Prag S, Adams JC: Molecular phylogeny of the kelch-repeat superfamily reveals an expansion of BTB/kelch proteins in animals. BMC Bioinformatics 4: 42, 2003

3. Ito N, Phillips SEV, Yadav KDS, Knowles PF: Crystal structure of a free radical enzyme, galactose oxidase. J Mol Biol 238: 794-814, 1994

4. Robinson DN, Cooley L: Drosophila kelch is an oligomeric ring canal actin organiser. J Cell Biol 138: 799-810, 1997

5. Adams JC, Kelso R, Cooley L: The kelch repeat superfamily: propellers of cell function. Trends Cell Biol 10, 17-24, 2000

6. Wilson AC, LaMarco K, Peterson MG, Herr W: The VP16 accessory protein HCF is a family of polypeptides processed from a large precursor protein. Cell 74: 115-125, 1993

7. Kristie TM, Sharp PA: Purification of the cellular $\mathrm{C} 1$ factor required for the stable recognition of the Oct-1 homeodomain by the herpes simplex virus $\alpha$-transinduction factor (VP16). J Biol Chem 268: 6525-6534, 1993

8. Lu R, Yang P, O'Hare P, Misra V: Luman, a new member of the CREB/ATF family, binds to herpes simplex virus VP16-associated host cell factor. Mol Cell Biol 17: $5117-5126,1997$

9. Freiman RN, Herr W: Viral mimicry: common mode of association with HCF by VP16 and the cellular protein LZIP. Genes Dev 11, 3122-3127, 1997 
10. Vogel JL, Kristie TM: The novel coactivator C1 (HCF) coordinates multiprotein enhancer formation and mediates transcription activation by GABP. EMBO J 19, $683-669,2000$

11. Wilson AC, Boutros M, Johnson KM, Herr W: HCF-1 amino- and carboxyterminal subunit association through two separate sets of interaction modules: involvement of fibronectin type 3 repeats. Mol Cell Biol 20: 6721-6730, 2000

12. Wysocka J, Myers MP, Laherty CD, Eisenman RN, Herr W: Human Sin3 deacetylase and trithorax-related Set1/Ash2 histone H3-K4 methyltransferase are tethered together selectively by the cell-proliferation factor HCF-1. Genes Dev 17: 896-911, 2003

13. Wysocka J, Reilly PT, Herr W: Loss of HCF-1-chromatin association precedes temperature-induced growth arrest of tsBN67 cells. Mol Cell Biol 21: 3820-3829, 2001

14. Julien E, Herr W: A switch in mitotic histone H4 lysine 20 methylation status is linked to M phase defects upon loss of HCF-1. Mol Cell 14: 713-725, 2004

15. Zhou HJ, Wong CM, Chen JH, Qiang BQ, Yuan JG, Jin DY: Inhibition of LZIPmediated transcription through direct interaction with a novel host cell factor-like protein. J Biol Chem 276: 28933-28938, 2001

16. Diaz E, Schimmoller F, Pfeffer SR: A novel Rab9 effector required for endosometo-TGN transport. J Cell Biol 138: 283-290, 1997

17. Jin DY, Wang HL, Zhou Y, Chun ACS, Kibler KV, Hou YD, Kung H, Jeang KT: Hepatitis C virus core protein-induced loss of LZIP function correlates with cellular transformation. EMBO J 19: 729-740, 2000 
18. Chin KT, Zhou HJ, Wong CM, Lee JMF, Chan CP, Qiang BQ, Yuan JG, Ng IOL, Jin DY: The liver-enriched transcription factor CREB-H is a growth suppressor protein underexpressed in hepatocellular carcinoma. Nucl Acids Res 33: 1859-1873, 2005

19. apRhys CMJ, Ciufo DM, O'Neill EA, Kelly TJ, Hayward GS: Overlapping octamer and TAATGARAT motifs in the VF65-response elements in herpes simplex virus immediate-early promoters represent independent binding sites for cellular nuclear factor III. J Virol 63: 2798-2812, 1989

20. Zhou Y, Kok KH, Chun ACS, Wong CM, Wu HW, Lin MCM, Fung PCW, Kung H, Jin DY: Mouse peroxiredoxin $\mathrm{V}$ is a thioredoxin peroxidase that inhibits p53induced apoptosis. Biochem Biophys Res Commun 268: 921-927, 2000

21. Zhou Y, Ching YP, Ng RWM, Jin DY: Differential expression, localization and activity of two alternatively spliced isoforms of human APC regulator CDH1. Biochem J 374: 349-358, 2003

22. Siu YT, Chin KT, Siu KL, Choy EYW, Jeang KT, Jin DY: TORC1 and TORC2 coactivators are required for Tax activation of the human T-cell leukemia virus type 1 long terminal repeats. J Virol 80: 7052-7059, 2006

23. Zhou Y, Ching YP, Chun ACS, Jin DY: Nuclear localization of the cell cycle regulator CDH1 and its regulation by phosphorylation. J Biol Chem 278: 1253012536,2003

24. Ching YP, Chan SF, Jeang KT, Jin DY: Retroviral oncoprotein Tax targets coiledcoil centrosomal protein TAX1BP2 to induce centrosome overduplication. Nat Cell Biol 8: 717-724, 2006 
25. Chen JH, Luo WQ, Zhou Y, Tan XY, Li GT, Huang XW, Zhou HJ, Li WJ, Yuan JG, Qiang BQ: cDNA cloning and expression in E. coli of a human new gene HCL. Prog Nat Sci 10: 1001-1005, 2000

26. Jin DY, Spencer F, Jeang KT: Human T cell leukemia virus type 1 oncoprotein Tax targets the human mitotic checkpoint protein MAD1. Cell 93: 81-91, 1998

27. Yedowitz JC, Kotsakis A, Schlegel EF, Blaho JA: Nuclear localizations of the herpes simplex virus type 1 tegument proteins VP13/14, vhs, and VP16 precede VP22-dependent microtubule reorganization and VP22 nuclear import. J Virol 79: 4730-4743, 2005

28. Chida K, Nagamori S, Kuroki T: Nuclear translocation of Fos is stimulated by interaction with Jun through the leucine zipper. Cell Mol Life Sci 55: 297-302, 1999

29. Gettemans J, Meerschaert K, Vandekerckhove J, De Corte V: A kelch $\beta$ propeller featuring as a G $\beta$ structural mimic: reinventing the wheel? Sci. STKE 191: PE27, 2003

30. Vogel JL, Kristie TM: Autocatalytic proteolysis of the transcription factor coactivator $\mathrm{Cl}(\mathrm{HCF})$ : a potential role for proteolytic regulation of coactivator function. Proc Natl Acad Sci USA 97: 9425-9430, 2000

31. Julien E, Herr W: Proteolytic processing is necessary to separate and ensure proper cell growth and cytokinesis functions of HCF-1. EMBO J 22, 2360-2369, 2003

32. Johnson KM, Mahajan SS, Wilson AC: Herpes simplex virus transactivator VP16 discriminates between HCF-1 and a novel family member, HCF-2. J Virol 73: 39303940,1999 
33. Lee $\mathrm{S}$, Herr $\mathrm{W}$ : Stabilization but not the transcriptional activity of herpes simplex virus VP16-induced complexes is evolutionarily conserved among HCF family members. J Virol 75: 12402-12411, 2001

34. Raggo C, Rapin N, Stirling J, Gobeil P, Smith-Windsor E, O'Hare P, Misra V: Luman, the cellular counterpart of herpes simplex virus VP16, is processed by regulated intramembrane proteolysis. Mol Cell Biol 22: 5639-5649, 2002

35. Kondo S, Murakami T, Tatsumi K, Ogata M, Kanemoto S, Otori K, Iseki K, Wanaka A, Imaizumi K: OASIS, a CREB/ATF-family member, modulates UPR signalling in astrocytes. Nat Cell Biol 7: 186-194, 2005.

36. Sadowski I, Ma J, Triezenberg SJ, Ptashne M: GAL4-VP16 is an unusually potent transcriptional activator. Nature 335: 563-564, 1988

37. Baichwal VR, Park A, Tjian R: v-Src and EJ Ras alleviate repression of c-Jun by a cell-specific inhibitor. Nature 352: 165-168, 1991 


\section{Figure legends}

Fig. 1. Sequence alignment of representative vertebrate KLHDC1 and KLHDC2 proteins. Perfect matches and conservative substitutions were boxed in black and gray, respectively. Human HCF-1 sequence (HCFC1) was included for comparison and the start positions of the six kelch repeats were also highlighted. GenBank identification numbers (gi) are as follows: human KLHDC1, 28380069; mouse Klhdc1, 30142701; pufferfish klhdc1, 47229997; human KLHDC2, 7657301; human HCFC1, 4885403.

Fig. 2. Phylogenetic tree of vertebrate KLHDC1-/KLHDC2-related proteins. Phylogeny was inferred from protein sequences aligned with the PILEUP program in the Wisconsin package using parsimony computed with the PROTPARS program in the PHYLIP package. SEQBOOT and CONSENSUS programs in the same package were used to perform bootstrap replication and to generate the majority rule consensus tree from 100 replicates. Numbers on the nodes are bootstrap confidence probabilities (\%). Human kelch repeat protein p40 was used as an outgroup in this tree. GenBank identification numbers (gi) are as follows: chicken Klhdc1, 50748920; mouse Klhdc1, 30142701; rat Klhdc1, 62650770; human KLHDC1, 28380069; pufferfish klhdc1, 47229997; chicken Klhdc2, 60302820; mouse Klhdc2, 13542753; rat Klhdc2, 34865313; human KLHDC2, 7657301; pufferfish klhdc2, 47229999; human p40, 33695109.

Fig. 3. Genomic organization of vertebrate KLHDC1 and KLHDC2 loci. A. Schematic delineation of human KLHDC1 and KLHDC2 at chromosome 14q21.3. Arrows indicate orientation of transcription. Map locations were based on human genome sequence Build 
35.1. POLE2, DNA polymerase $\varepsilon 2$ (p59 subunit); SDCCAG1, serologically defined colon cancer antigen 1; Tel, telomeric; Cen, centromeric. B. Schematic diagram of mouse Klhdc1 and Klhdc2 at chromosome 12. Map locations were based on mouse genome sequence Build 34.1. C. Schematic delineation of pufferfish klhdc1 and klhdc2 at chromosome 10. Map locations were based on pufferfish genome sequence provided by Genoscope, the French National Sequencing Center (http://www.genoscope.cns.fr). D. Comparison of the genomic structure of human $(\mathrm{H})$, mouse $(\mathrm{M})$ and pufferfish $(\mathrm{P})$ KLHDC1 locus. The sizes of 13 exons are shown in nucleotides. E. Comparison of the genomic structure of human $(\mathrm{H})$, mouse $(\mathrm{M})$ and pufferfish $(\mathrm{P})$ KLHDC2 locus. The sizes of 12 (pufferfish) or 13 (human and mouse) exons are shown in nucleotides.

Fig. 4. Northern blot analysis of KLHDC1 (A) and KLHDC2 (B) mRNA in human tissues. KLHDC1- and KLHDC2-specific probes hybridized to mRNA species migrating at $2.7 \mathrm{~kb}$ and $2 \mathrm{~kb}$, respectively. The same blot was first probed for KLHDC1, stripped, and then re-probed for KLHDC2. $\beta$-actin-specific hybridization was performed on this blot after stripping the KLHDC2 signal. Blots were from CLONTECH and all lanes contain $\sim 2 \mu \mathrm{g}$ of polyadenylated RNA. KLHDC1 and KLHDC2 transcripts are indicated. PBL: peripheral blood leukocytes.

Fig. 5. Differential activity of KLHDC2 and KLHDC1 in the inhibition of LZIPmediated transcription. HeLa cells were transfected with pGal-Luc reporter plasmid and expression vectors for the indicated proteins. Expression of the V5-tagged KLHDC1 and KLHDC2 proteins in transfected cells was verified by Western blotting. Results are 
representative of three independent experiments and the error bars indicate S.E. Luc activity: relative firefly luciferase activity in arbitrary units normalized to sea pansy luciferase activity.

Fig. 6. Subcellular localizations of KLHDC2 and KLHDC1 in HeLa cells. pcDNAKLHDC2 (panels A-C), pcDNA-KLHDC1 (panels D-F), pBD-VP16-KLHDC1 (panels G-I) and pBD-cJun-KLHDC1 plasmids were individually transfected into HeLa cells. Cells were fixed and stained for KLHDC1/2 with mouse monoclonal anti-V5 ( $\alpha$-V5; panels $\mathrm{A}$ and $\mathrm{D})$ or anti-BD ( $\alpha-\mathrm{BD}$; panels $\mathrm{G}$ and $\mathrm{J})$ antibody. Nuclear morphology was visualized by counterstaining with propidium iodide (PI; panels B, E, H and K). Green (KLHDC1/2) and red (PI) fluorescent signals were overlaid by computer assistance (panels C, F, I and L). Co-localizations are in yellow. The same fields of cells are shown in panels A-C, D-F, G-I and J-L. Results represent three independent experiments and 100 transfected cells from each experiment were quantitated. While $88 \pm 5 \%$ of KLHDC1-expressing cells showed predominantly cytoplasmic staining of KLHDC1, 86 $\pm 7 \%$ of KLHDC2-positive cells showed predominantly nuclear staining of KLHDC2. The nuclear localization of BD-VP16 and BD-c-Jun has been shown elsewhere [36, 37] and verified in our experiment (data not shown).

Fig. 7. Subcellular localization of KLHDC2 and KLHDC1 in HeLa cells treated with leptomycin B (LMB). Cells were transfected with either pcDNA-KLHDC2 (panels A-F) or pcDNA-KLHDC1 (panels G-L). LMB was added to a final concentration of $15 \mathrm{nM}$ 
and cells were incubated for additional 5 hours (panels D-F and J-L). Immunostaining was then performed as in Fig. 5.

Fig. 8. Neither KLHDC2 nor KLHDC1 associates with actin. MCF-7 cells grown to about $70 \%$ confluence in 10-cm dishes were either mock transfected (lanes 1 and 2) or transfected with expression plasmid for V5-tagged $\beta$-galactosidase (lanes 3 and 4), KLHDC2 (lanes 5 and 6) or KLHDC2 (lanes 7 and 8). Twenty-four hours after transfection, cells were lysed in HEPES lysis buffer and immunoprecipitated with rabbit polyclonal anti-V5. The protein complexes were subsequently resolved in 10\% SDSPAGE followed by Western blotting using mouse anti-actin (A). Western blot analysis with mouse anti-V5 was also performed to detect V5-tagged proteins in the immunoprecipitates (B). Lanes 1, 3, 5 and 7 contain 1/10 input materials, while the immunoprecipitates (IP) are in lanes 2, 4, 6 and 8 . Asterisk $(*)$ indicates the heavy chain of $\operatorname{IgG}$. 
Repeat 1 ------- MQY EPVMDPGFQGHFSELVAR--ERSGHTAVVEGHRLYVWGGYMSVADD-- -EVFLPNDEIW
-MADGNEDLRADDLPGPAFESYESMELACPAERSGHVAVSDGRHMFVWGGYKSNOVRGLYDFYLPREELW

1 MAS AVS PANLPAVLLQPRWKRVVGWSGPVPRPRHG HRAVA I KE L IVVFGGGNE-- - - - - - - GIVDELH

\section{Repeat $2 \longrightarrow$}

48 TYD IDSGLWTRMLMEGELPASMSGSCGACINGKLYIFGGYDDKG-Y

48 TYDIDSGLWKMHLMEGELPPSMS GSCGACI HGRLYVEGGYDDKG

58 AYDLNRGVWEVFHMTGDTP P SMS GTCGCSVNGHMY I FGGCDDNG

Y S NRLY FVNLRTRDETYIWEKI TDF

70 IYNMETGRWKKINTEGDVP P SMS GSCAVCVDRVLYLFGGHHSRG

Y SNRLY FVNLRTRD GTYTWEKI TKE

NTNKFYMLDSRSTDRVLQWERID - C

61 VYNTATNQWF I PAVRGDIP PGCAAYGFVCDGTRLLVFGGMVEYGKY SNDLYELQASRWEWKRLKAKTP-K

Repeat $3 \longrightarrow$

117 EGQPPTPRDKLSCWVYKDRLIY FGGYGCRRH SELQD -

117 DGQPPTPRDKLSCWVYKDRLI Y FGGYGYRRH SELQE-

CFDVHDAS-WEEQIFWGWHNDVH I FDTKTQT

138 QGI PPSS KDKLGVWVYKNKLIFF GGYGYLPEDKVLG - - TEEFDETSFWNS S PRGWNDHVH I LDTETT 130 NGPPPCPRLGHSF SLVGNKCYLFGGLANDSEDPKNN- - - - - - - - - - - - - - I RYLNDLYILELRPGS

\section{Repeat $4 \longrightarrow$}

183 - - - - WFQPEIKGGVPPQPRAAHTCAVLGNKG- - - -YIFGGRVLQTRMNDLHYLNLDTWTWSGRITINGE

183 - - - -WSOPEIKGGVPPOPRAAH SCAVIGNKG- - - - YVFGGRVLOTRMNDLHYLNLDTWVWSGRISVNGE

194 - - - WSEPKTNG-RAPAPRAAHA SATLGHRG - - - - Y I CGGRVMETRT SDVHCIDFETWTWT-EITPASP

$205----W S Q P I T T$ T-KAPSPRAAHACATVGNRG- - - - FVFGGRYRDARMNDIHYLNLDTWEWN-ELIPQGI 183 GVVAWDIPITYG-VLPPPRESHTAVVYTEKDNKKS KLVI YGGMSGCRLGDLWTLD IDTLTWN-KPSL SGV

Repeat $5 \longrightarrow$

Repeat $6 \longrightarrow$

244 SPKHRSWHTLTPIADDKLFLCGGLSADN I PL SDGWI HNVTTNCWKQLTHLPKT- - RPRLWHTACLGKENE

244 S PKHRSWHTLTAITDDKLF LFGGLNADN I PL SDGWI HNITTNCWKQLRH LPYT- - RPRLWH TACLGKENE

253 VPVGRSWHTLTAVSDHSLFLFGGLSVDCNPMSDGWLLDVEKKTWREVDHPFKN- KPRLWHTACQGRDAD 264 CPVGRSWHSLTPVSSDHLFLFGGFTTDKQPL SDAWTYCI S KNEWIQFNHPYTE - KPRLWHTACASDEGE 251 APLPRSLHSATTIG-NKMYVFGGWVP---LVMDDVKVATHEKEWKCTNTLACLNLDTMAWETI LMDTLED

312 IMVEGGSKDDLLALD-TGHCNDLIIFQTQPY SLLR SCLDC I GK- - -NS IMLESQISLLPPKLLQQVLKKI

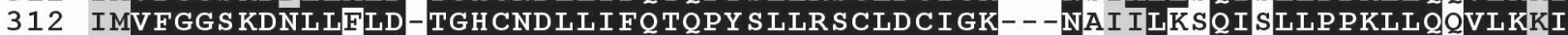
321 VIVFGGSCDY ILLVD-TGH CNDALVFQTQPYPLFRICEDYIAKNVKNYEALRNQLPRLPSKLITAVQARM 332 VIVEGGC ANNLLVH RAAHSNEI L FS VQPKS LVRL SLEAVIC - - - F KEIANSWNCLPKHLLH SVNQRF 317 NIPRARAGHCAVAIN---- - TRIYIWS GRD-GYRKAWNNQVCCKDLWYLETEKPP P ARVQLVRANTNSL

378 TFWAAANHREEQRVQKEETENKYQWIS SN 378 TFWTAANYRKEQRIRKEETENNQPRVS SC 390 CFY----------------------399

381
GSNNTSGS

EVSWGAVATA-----------------
KLHDC1-human

KIhdc1-mouse

K1hdc1-pufferfish

KLHDC 2 - human

HCEC1-human 


\section{POLE2 KLHDC1 KLHDC2 SDCCAG1}

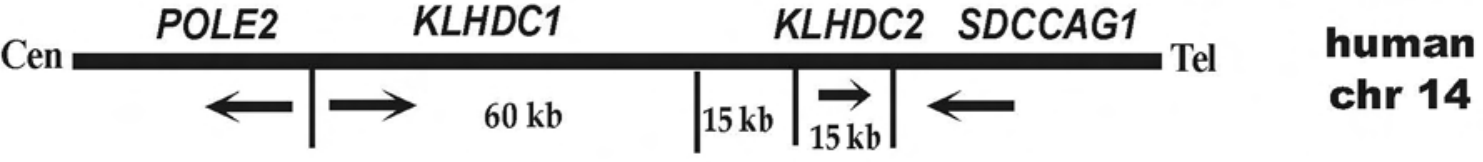

B

Cen

Pole2 KIhdc1

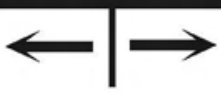

$42 \mathrm{~kb}$
KIhdc2 Sdccag1

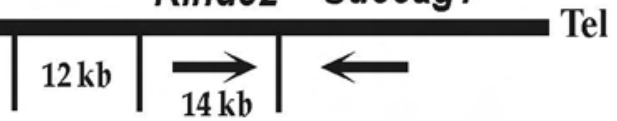

mouse

chr 12

\begin{tabular}{r|c|c|c|c|c|c|} 
Cen sdccag1 & \multicolumn{1}{c}{ klhdc2 } & pole2 & klhdc1 & Tel $\begin{array}{c}\text { pufferfish } \\
\text { chr 10 }\end{array}$
\end{tabular}

D

\begin{tabular}{|c|c|c|c|c|c|c|c|c|c|c|c|c|c|}
\hline & 1 & 2 & 3 & 4 & 5 & 6 & 7 & 8 & 9 & 10 & 11 & 12 & 13 \\
\hline $\mathrm{H}$ & 124 & 71 & 118 & 119 & 79 & 84 & 84 & 59 & 112 & 73 & 85 & 53 & 1564 \\
\hline $\mathbf{M}$ & 182 & 71 & 118 & 119 & 79 & 84 & 84 & 59 & 112 & 73 & 85 & 53 & 829 \\
\hline$P$ & 117 & 71 & 118 & 113 & 88 & 84 & 81 & 59 & 110 & 73 & 85 & 53 & 373 \\
\hline
\end{tabular}

$\mathbf{E}$

\begin{tabular}{|c|c|c|c|c|c|c|c|c|c|c|c|c|c|}
\hline & 1 & 2 & 3 & 4 & 5 & 6 & 7 & 8 & 9 & 10 & 11 & 12 & 13 \\
\hline $\mathrm{H}$ & 469 & 80 & 118 & 116 & 82 & 84 & 81 & 59 & 110 & 73 & 85 & 53 & 308 \\
\hline $\mathbf{M}$ & 380 & 80 & 118 & 116 & 82 & 84 & 81 & 59 & 112 & 73 & 85 & 53 & 312 \\
\hline $\mathrm{P}$ & 141 & 80 & 118 & 113 & 256 & 81 & 59 & 110 & 76 & 88 & 53 & 124 & \\
\hline
\end{tabular}




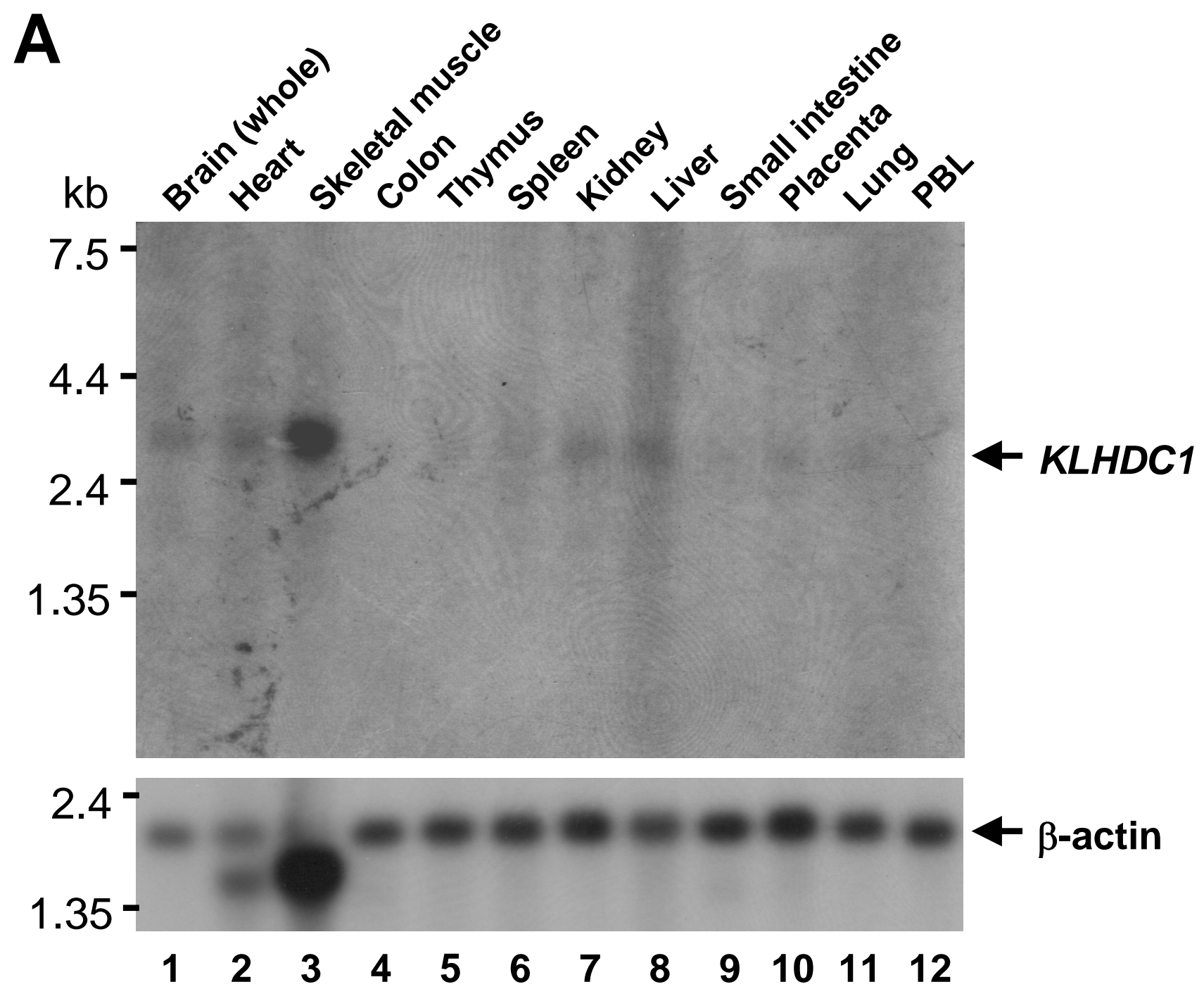

Figure 4A 


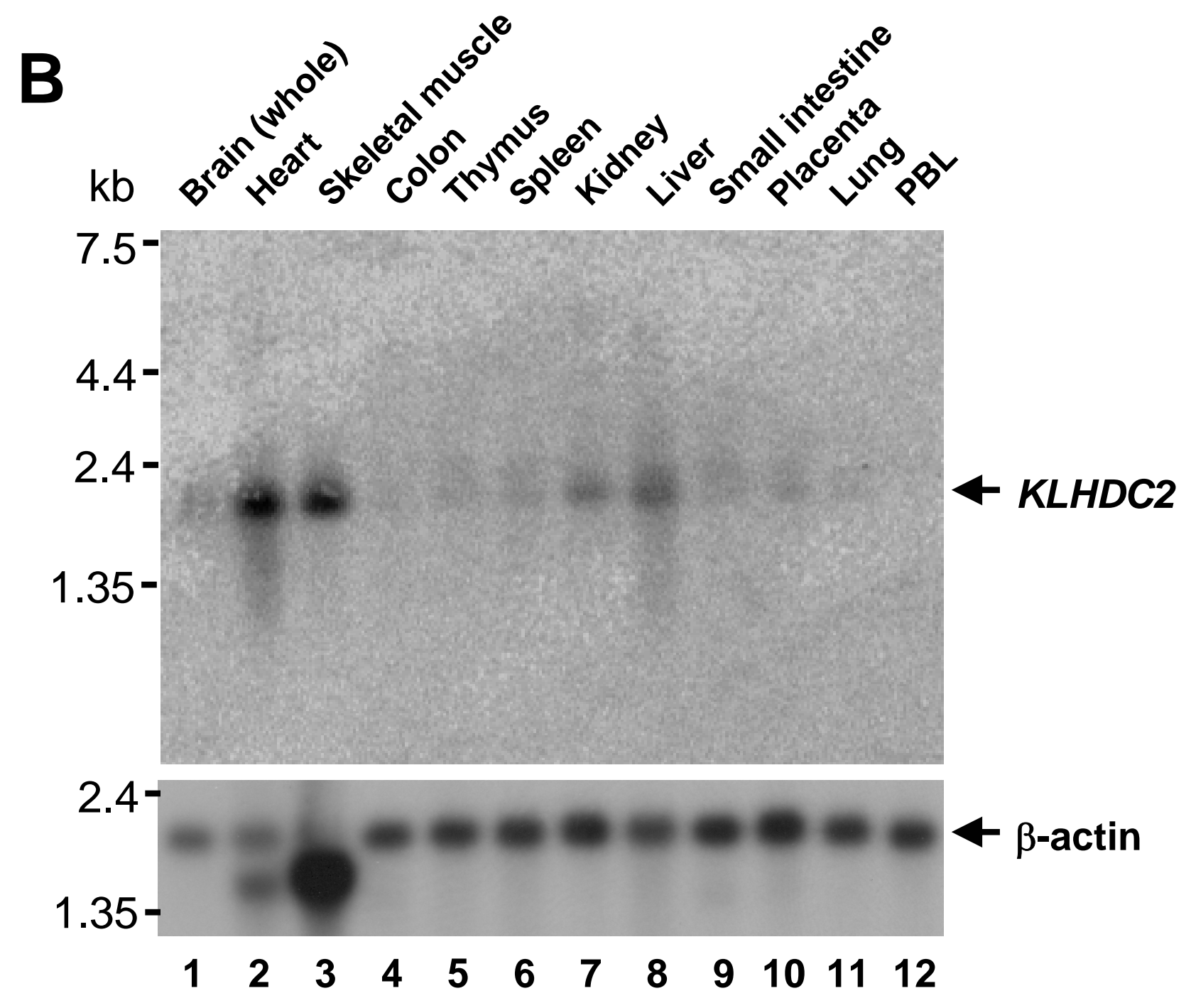

Figure 4B 


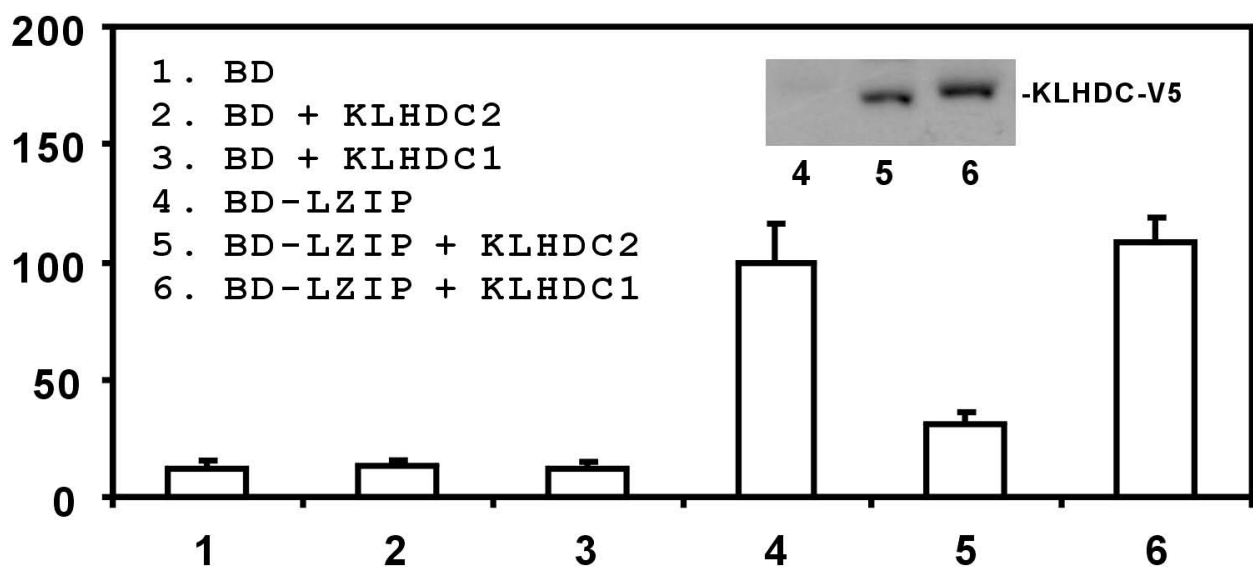



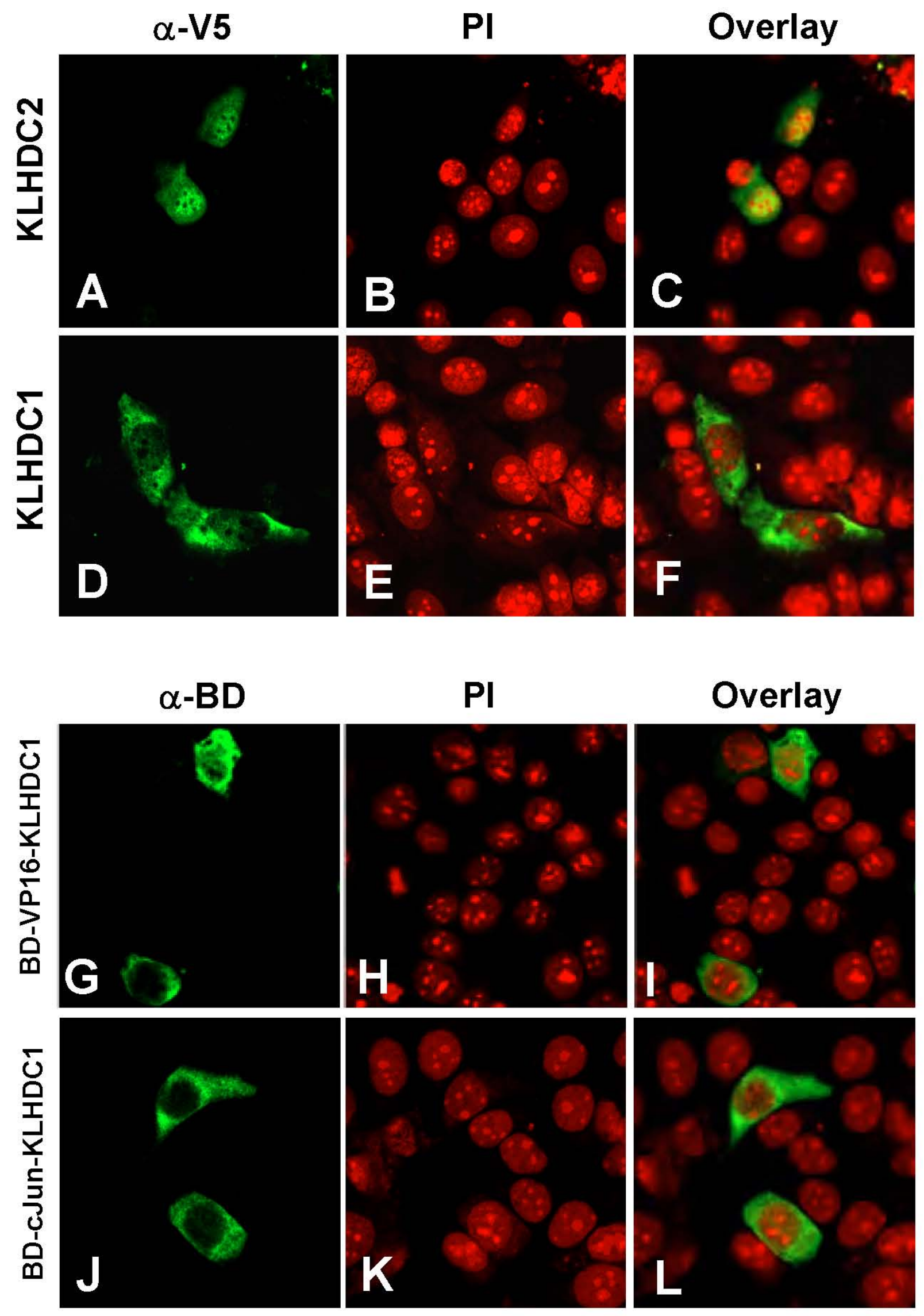

Figure 6 


\section{KLHDC2}

A

B

C

\section{KLHDC2}

D

E

F

G

H

I

KLHDC1

J

K

L

Figure 7 


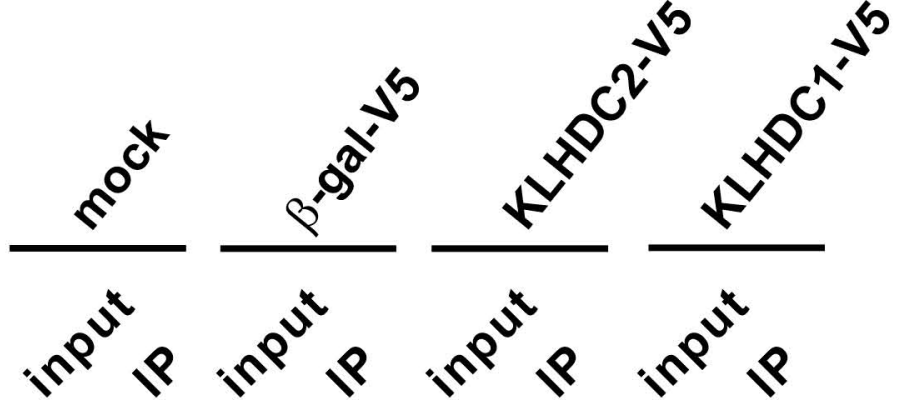

(kDa)

55

40

B

$100-$

72

55

$40-$

4 KLHDC-V5

$\begin{array}{llllllll}1 & 2 & 3 & 4 & 5 & 6 & 7 & 8\end{array}$ 